\title{
The universe is expanding by entirely moving at some very high speed and not by flying away of the galaxies from each other
}

\author{
Salah Abunaieb \\ Kalba power and desalination station, Sharjah Electricity and Water Authority, U.A.E \\ E-mail: salnayib@hotmail.com
}

\begin{abstract}
Expansion of the universe has already been confirmed by the Hubble discovered nebular red-shift. But, is it expanding in the simple Newtonian way that the distance between any observed distant star and the observer is continuously increasing? The argument against this is: If so, then the apparent brightness and size of that distant star will continuously decrease and thus the stars at the outer edge of the visible universe will disappear in no time. To understand the way in which the universe is expanding it is important, in accordance with the special theory of relativity, to bear in mind the fundamental difference between the way electromagnetic waves spread and the way Newtonian point particles move. Accordingly, the Hubble discovered nebular red-shift is explained here as due to relative motion between the Newtonian point particle observer, which is moving along with the entire universe, and the space-time fixed origins (Events) of the received pulses of light. But, as the source is also moving with the entire universe, these space-time fixed origins are continuously repeated on the continuous world line of the star and hence the recession is continuously repeated while the star remains at the same fixed distance from the observer.
\end{abstract}

Keywords: Expansion; Universe; Galaxies; Nebular Red-Shift; Stars.

\section{Introduction}

The idea that the universe is expanding dates back to 1826 , when it was perversely demonstrated by Olbers' paradox. Simply, the paradox of the German astronomer Wilhelm Olbers consisted of three very reasonable assumptions. The first was that the universe consisted of concentric layers of stars that added up one to another without limit. The second assumption was the symmetrical dispersal of stars in all regions of the universe. The third was that the universe was static. But, Olbers then found that the sum of light received on Earth from these layers of stars stretching out to infinity would give an incandescent glow theoretically equal to 50000 times the light from the sun at its zenith. This meant that there should be no night and that temperature on the Earth should go up to $5500^{\circ} \mathrm{C}$. Being that simple and of such reasonable assumptions the paradox was so significant and inescapable. But its conclusions were not factual. In fact there is, of course, night and day and the maximum temperature on Earth is around $50^{\circ} \mathrm{C}$ only. Olbers' assumptions were, hence, re-examined and finally the dilemma was found to lie in the assumption of a static universe which was, hence, reversed to the assumption of an expanding universe. Because in an expanding universe, unlike in a static one, the intensity of light we receive from the receding stars will obviously be reduced. In this way Olbers' paradox was escaped and expansion of the universe was thus perversely demonstrated (Inc. Staff Encyclopaedia Britannica Publishers 1979).

Taking into account the observed uniformity of the universe, the expansion theory was faced in the beginning by the question of how the universe could maintain this uniformity while moving.
The only one possible answer was that the velocity of every object should be in the line of sight and proportional to its distance (Inc. Staff Encyclopaedia Britannica Publishers 1979).

Anyhow, the proof that the universe is expanding and in the way that maintains the observed uniformity came some hundred years later. That was when the American astronomer Edwin Hubble, confirmed the existence of galaxies other than our parent one in 1924. Hubble then started to work out the distances to these new galaxies and observe their light spectra. He found that nearly all of these extremely distant galaxies were of red-shifted light spectra with the size of the red-shift proportional to the galaxy's distance. By the Doppler Effect this was explained as a recession of every observed galaxy at a velocity proportional to its distance from us (Hawking 1995).

Now, this confirmed expansion and in the way explained above was learned from the waves of light we received from distant galaxies. As per the kinematic postulate of the special theory of relativity of Albert Einstein, the speed of light in a vacuum has the same value relative to all inertial reference frames and is independent of the relative velocity of the light source and the observer (Skinner 1982). Skinner reported that Einstein's kinematic postulate, being consistent with all experimental results, has achieved the status of a law of physics (Skinner 1982). In view of this law of physics, it is shown in this study that the conclusion in the sense that the distance between the observer and the observed object is increasing with time is not the only one explanation of Hubble's findings. The same findings can be alternatively explained when the universe is entirely moving at some very high speed while the distances are constant. This alternative explanation is not, however, contradicted with the following simple argu- 
ment that contradicts with the present conclusion that the distance between the observer and the observed object is continuously increasing.

\section{Argument}

If expansion of the universe is so that the distance between the observer and the observed object is continuously increasing then the apparent brightness and the apparent size of that object will obviously decrease with time. Thus, the stars at the edge of visibility (which are hence of the highest recession velocities in the visible universe) would disappear in no time if they were really flying away. In a symmetrical universe that consists of concentric layers of stars that add up without limit it is sure that every night there will be some stars which are just at the edge of visibility and these would disappear in no time if they were really flying away at such fantastic speeds.

\section{Assumptions}

While accepting Olbers' first and second assumptions, the third assumption (that the universe is static) and its revised form (the Newtonian expanding universe) are both modified as follows: The universe is entirely moving at some very high speed. Due to this motion, as it will be seen, the Hubble discovered nebular redshift can be explained as recession that is continuously repeated while the observed object remains at the same relative position and the same constant distance from the observer.

\section{Justification of the assumption that the uni- verse is entirely moving}

It is very long time now, since the static state was thought to be natural and since the time when it was the duty of those who thought otherwise to prove it for their relaxed static opponents. Like that and in one step the assumption that the universe is entirely moving is at least $50 \%$ reasonable. But when we think of the many different ways we are moving in this universe we will tend to believe that the assumption is more than just $50 \%$ reasonable Now and as per the data given by Mayas, it can be estimated that one is moving at about $0.4 \mathrm{~km} / \mathrm{sec}$ around the axis of the Earth, at about $30 \mathrm{~km} / \mathrm{sec}$ along with the Earth and the Moon around the Sun and at about $285 \mathrm{~km} / \mathrm{sec}$ along with the entire Solar System around the centre of the Milky Way (Mayas 1999). Well, now we are only one or two steps away from saying: and at the speed of so and so along with the entire universe. So the assumption is very reasonable. Later in the discussion, anyhow, we will come across more than one known and confirmed observation in support of this assumption.

\section{Analogy of the escape from Olbers' paradox in the entirely moving universe}

As mentioned in the introduction it was the escape from the in candescent glow of Olbers' paradox that was behind the theories of the expanding universe. The analogy to this escape in the entirely moving universe is the escape of racing cars from the dust that they raise. Let us think of a Desert Car Race where all the racing cars start from one start line and at the same time, speed and in the same direction. Assuming no wind, the dust raised by the cars will slowly spread in air. With respect to the champs the visibility is not affected by this dust as far as they keep moving at the same speed and in same direction. If any one car stops or slows down then it will be covered with the dust from all others. Of course there is no comparison between the speed of light and the speed of dust spread in the air. The point of comparison is that the dust spreads in air and in all directions (assuming no wind) from relatively fixed points on the racing tracks and independent of the speed of the cars. The moving cars, thus, escape the dust they raise. In the entirely moving universe and as per the special theory of relativity, any emitted pulse of light will spread out spherically at the speed of light from an absolutely fixed point in space-time and independent of the speed of the source (Inc. Staff Encyclopaedia Britannica Publishers 1979, Skinner 1982, Hawking 1995). Now, let us consider an extended source of light passing across a fixed point in space over some period of time. That point will be a space fixed origin of concentric waves of light emitted during that period of time. While receiving the same set of concentric waves, the observer in the entirely moving universe will be flying away from their fixed origin as will be explained in the following discussion.

\section{Moving universe and the frequency of light}

Hawking, while explaining the Doppler Effect, stated the following: "Now imagine a source of light at a constant distance from us, such as a star, emitting waves of light at a constant frequency. Obviously the frequency of the waves we receive will be the same as the frequency at which they are emitted." (Hawking 1995, p. 23-24). Well, we will see now that this commonly believed statement is not generally true in a non-static universe. It is only conditionally true.

Actually the observation we make about distant objects is obtained from the waves of light (or any other electromagnetic waves) we receive at our very vicinity. But how waves are received is dependent on the distance of their origin. One obvious and wellknown example of the effect of the distance on our observation is the fact that when looking at any object then the more distant the smaller it appears. Also if the object is moving then the more distant the slower it appears to move or in other words the shorter the space it appears to travel. And that is why and how galaxies at fantastic distances appear as single tiny stars which are fixed in position relative to each other. Hence, with respect to the observer, the distance shortens the space (whether it is dimensional space of objects or space travelled by them). But it must be noted that the space that is shortened by the distance is the space normal to the line of sight.

Like that when looking at a star in the sky then, proportional to distance, one can think of some space around and normal to the line of sight which is apparently reduced to only the thickness of that line of sight. The constant of proportionality is some very infinitesimally small non-zero angle $(\alpha)$ as shown in Figure 1.

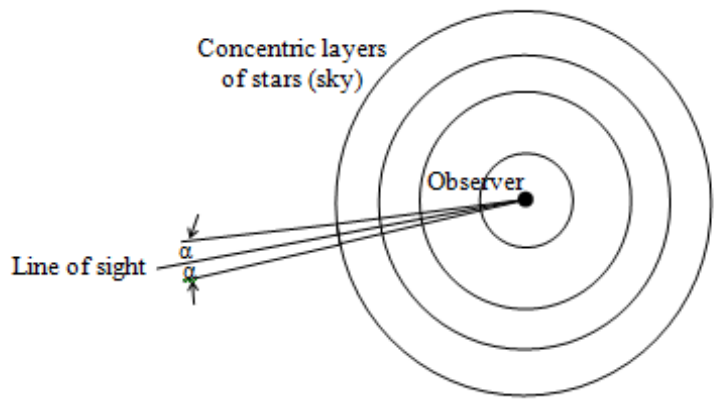

Fig. 1: Drawn by Author

This space, which is apparently that small, is termed here the point star. So, the real radius of the point star is proportional to the distance from the observer and its surface is assumed plane, even continuous and steady. Here, steady means that it is not a fading out star.

Now let us consider a point star and a distant point particle observer moving at the same velocity $\mathrm{V}$ along with the entire universe. The effect of motion on the frequency of the received light waves is then discussed for the following three different cases. 


\subsection{When the velocity is perpendicular to the initial line of sight}

Now both the observer and the point star are moving at the same velocity $\mathrm{V}$ along with the entire universe in a direction perpendicular to the initial line of sight as shown below in Figure 2.

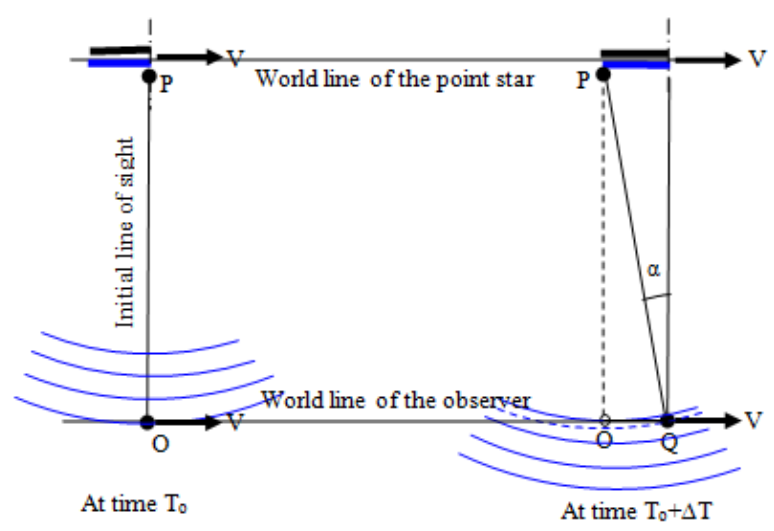

Fig. 2: Drawn by Author

Let point $\mathrm{O}$ be a space-fixed point at which the point observer receives the first pulse of light emitted from the space-fixed point $\mathrm{P}$ when just reached at by the point star. Starting from point $\mathrm{O}$ at time $\mathrm{T}_{0}$, the moving point observer will continue to receive concentric waves of light from the space-fixed point $\mathrm{P}$ while the plain, even, continuous and steady surface of the point star is passing across it. The space-fixed point $\mathrm{P}$ will stay within the same point star i.e. within the same line of sight (relative to the observer) until its corresponding relative angular displacement is equal to the constant angle $\alpha$ at time $\mathrm{T}_{0}+\Delta \mathrm{T}$. Evidently, in this case, the frequency of the waves received is less than the frequency at which they were emitted from the space-fixed point $\mathrm{P}$ as the distance they travel to the observer is increasing from the minimum vertical (initial) distance PO to the maximum diagonal (final) distance PQ. As the world lines of the star and the observer are continuous lines then independent of the non-zero magnitude of the velocity $\mathrm{V}$ the shape in Figure2 is continuously repeated across the two paralle world lines. While moving (no matter how fast or slow), the very next instant a new point very next to point $\mathrm{P}$ is reached at by the point star. The pulse from this new point will be received via a new vertical line at a point very next to point $\mathrm{O}$ and at an instant very next to the instant of receiving the first pulse. So, this new point will recede in the same way from the minimum vertical to the maximum diagonal distance. At the back, the very next instant after point $\mathrm{P}$ is passed the just second last point will reach the maximum displacement and the pulse from it will be received at a point just next to point $\mathrm{Q}$. In between the minimum and maximum distant points, each space-fixed point will go to the very next further position (relative to the moving observer) on the plain, even, continuous and steady surface of the point star. On the other hand and as reported by Skinner, the eye records all the light that is received simultaneously, even though, if the object is extended, this light was emitted at different times from parts of the object at different distances from the observer (Skinner 1982). In the above case, hence, the observer will simultaneously receive all the pulses from all the space-fixed points the point star is simultaneously passing across. So, independent of the period of time $\Delta \mathrm{T}$ the recession can instantly be observed. Also, though moving relative to the space-fixed points on the world line of the point star there is no relative movement between the point particle observer and the point star which are moving at the same velocity V. Hence, the point star will remain at the same fixed distance from observer. In this way the recession is continuously repeated and can instantly be observed while the distance between the star and the observer is constant. So, there is no contradiction with the above mentioned argument as the apparent size and brightness of the star will remain the same and, no stars will disappear beyond the edge of visibility due to this mechanism of recession. By definition of the point star this recession is in the line of sight.

The above described movement is somewhat similar to the movement of a caterpillar vehicle on its continuous track. The vehicle moves on and relative to the part of the track that is firmly fixed on the ground. This fixed part of the track is continuously renewed (no matter how fast or slow the vehicle is moving) by continuously laying on the ground one segment at the front and picking up another at the back. Let us take the point particle observer, the point star and the initial and final lines of sight as a vehicle moving along the continuous world line of the star. This star-observer vehicle is moving on and relative to a continuous track of spacefixed origins of pulses of light. This continuous track of spacefixed origins is continuously renewed (independent of the nonzero magnitude of the velocity $\mathrm{V}$ ) by instantly reaching a new point at the front and leaving another at the back.

\subsection{When the velocity is in the line of sight}

Now both the point star and the observer are moving at the same velocity $\mathrm{V}$ which is in the line of sight. In this case it is only the distance that is affected as shown in Figure 3. The star will appear more distant than actual if the direction of the velocity $\mathrm{V}$ is from the star towards the observer and closer if in the opposite direction by an amount $(\Delta \mathrm{L})$ equal to the magnitude of the velocity $\mathrm{V}$ times the time taken by light to travel from the star to the moving observer in each case.

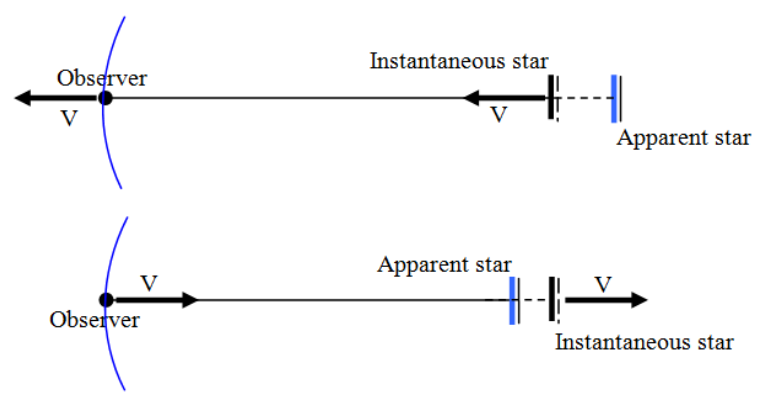

Fig. 3: Drawn by Author

In the first case: Let $\mathrm{L}$ be the actual distance between the instantaneous position of the star and the observer. At any instant of time light at the constant speed of light $\mathrm{c}$ will take a period of time $\Delta \mathrm{T}$ to travel from the apparent position of the star to the moving observer. But during the same period of time the star and the observer at the velocity $\mathrm{V}$ will both move a distance $\Delta \mathrm{L}$ away from where the light started at that instant of time i.e. from the apparent star position as shown in the following equation form:

$\Delta \mathrm{T}=\Delta \mathrm{L} / \mathrm{V}=(\mathrm{L}+\Delta \mathrm{L}) / \mathrm{c}$

From (1)

$\Delta \mathrm{L} / \mathrm{V}-\Delta \mathrm{L} / \mathrm{c}=\mathrm{L} / \mathrm{c}$.

From (2)

$\Delta \mathrm{L}=(\mathrm{V} / \mathrm{c}) \mathrm{L} /(1-\mathrm{V} / \mathrm{c})$.

In the second case: During a period of time $\Delta \mathrm{T}$ the distance travelled by both the star and the observer is $\Delta \mathrm{L}$, while the distance travelled by light is $(\mathrm{L}-\Delta \mathrm{L})$ and hence:

$\Delta \mathrm{L}=(\mathrm{V} / \mathrm{c}) \mathrm{L} /(1+\mathrm{V} / \mathrm{c})$.

The frequency is not affected as at equal intervals of time the origin of the successive pulses and the observer move equal distances. Each pulse will travel the same apparent distance to the moving observer. So, relative to the observer these pulses will be 
received as concentric waves of light the frequency of which is the same as the frequency at which they were emitted.

\subsection{When the velocity is in any arbitrary direction}

This is the general case which is simply the combination of the above two cases. It is when the direction of the velocity $\mathrm{V}$ makes an angle $\theta$ with the initial line of sight and on any plane around it as shown in Figure 4.
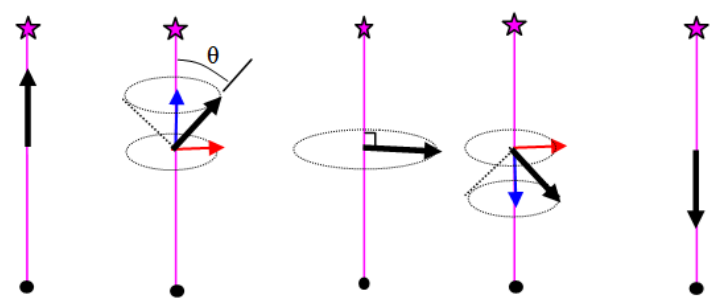

Fig. 4: Drawn by Author

The velocity $\mathrm{V}$ can then be analyzed into two vector components One component $(\mathrm{V} \cos \theta)$ will be in the line of sight and the other (V $\sin \theta$ ) perpendicular to the line of sight. So now, except when the velocity is exactly in the line of sight, there is always a nonzero perpendicular component of it. As shown above this perpendicular component will make the frequency of each concentric set of received light waves less than the frequency at which they were emitted from their relatively fixed centre. The other component (V $\cos \theta$ ) will affect the distance and at equal intervals of time will take both the observer and the origin of successive pulses of light equal distances along the initial line of sight as shown Figure 5.
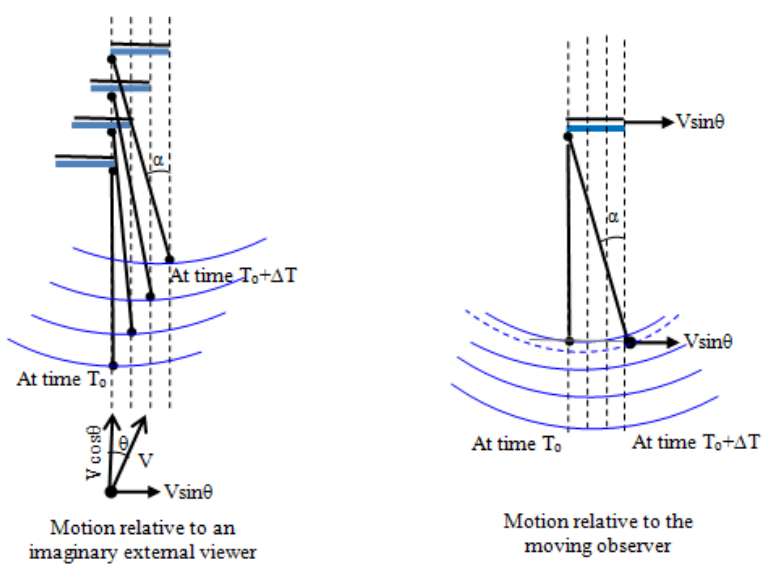

Fig. 5: Drawn by Author

Hence, we can now conclude from the above part of discussion the following: When a point star and an observer are moving at the same velocity while keeping a constant distance between them then, except when the velocity is exactly in the line of sight, the origins of the light received by the observer will continuously and repeatedly recede in the line of sight while the distance between the star and the observer remains constant.

\section{Recession as a function of time, distance and magnitude and direction of the velocity}

\subsection{Recession as a function of time}

It has already been shown that for an observer and a point star moving in parallel, the observer will simultaneously receive all the pulses from all the space-fixed points the point star is simultaneously passing across. So, independent of the period of time $\Delta \mathrm{T}$ the recession of all the points is instantly and simultaneously received. So, if the light spectrum of this star is checked then it will show red-shift of all the points from the minimum to the maximum distant on the plane, even, continuous and steady surface of the point star. Actually this method of finding the velocity of recession from the size of red-shift does not consider any period of time. It is known that Edwin Hubble did not take a stop watch in his hand to find out the velocity of recession. It was the Doppler Effect that helped Hubble to determine the velocity from the size of the redshift. The size of red-shift was found by comparing the position of some absorption lines on the light spectrum of the observed nebula to their relative position on the reference light spectrum of the sun. However, the rate of recession can still be theoretically found when only one single space-fixed origin of concentric waves of light is considered. As shown earlier any single space-fixed point the point star is passing across will recede from an initial minimum to a final maximum distance within the same point star. As diagrammatically exaggerated in Figure 6 below, it is clear that for equal intervals of time the recession rate of a single space-fixed point in the entirely moving universe is not constant. The recession rate is evidently speeding up.

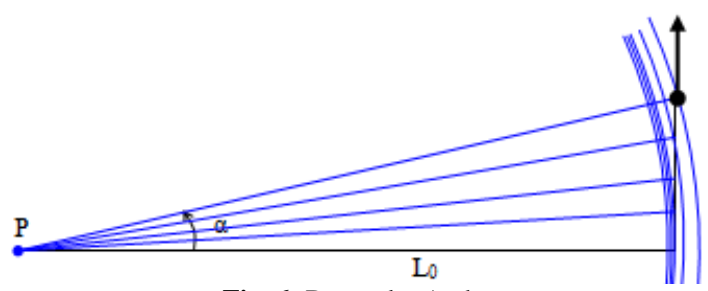

Fig. 6: Drawn by Author

The recent two findings that the universe is flat and that the recession is speeding up (Sincell 2001), can hence be explained as will be shown later.

\subsection{Recession as a function of the distance}

Now, as we have seen in above discussion, when a point star and an observer are moving at the same velocity in a direction perpendicular to the line of sight then any point the point star is passing across will recede from a minimum distance to a maximum distance within the same point star i.e. within the line of sight. Let $\mathrm{L}_{1}$ be the minimum and $\mathrm{L}_{2}$ the maximum distance as shown in Figure 7. The maximum amount of recession $(\Delta \mathrm{L})$ is then related to the distance $\mathrm{L}_{1}$ as follows:

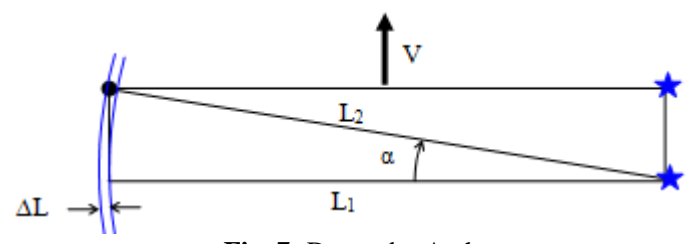

Fig. 7: Drawn by Author

$$
\Delta \mathrm{L}=\mathrm{L}_{2}-\mathrm{L}_{1}=\mathrm{L}_{1} \sec \alpha-\mathrm{L}_{1}=(\sec \alpha-1) \mathrm{L}_{1}
$$

Though infinitesimally small, the angle $\alpha$ is a non- zero constant. Hence the factor $(\sec \alpha-1)$ is a non-zero constant which is equal to (k), say. Hence,

$\Delta \mathrm{L}=\mathrm{k} \mathrm{L}_{1}$

Hence $\Delta \mathrm{L}$ is directly proportional to the distance $\mathrm{L}_{1}$

However, it must be noted that the distance $\mathrm{L}_{1}$ is the apparent distance. So, for objects at apparently equal distances the recession $\Delta \mathrm{L}$ will be the same.

\subsection{Recession as a function of the magnitude of velocity}

Though the recession is due to motion, its rate and value are independent of the magnitude of the velocity V. As shown earlier when the point star and the observer are moving at the same veloc- 
ity in a direction perpendicular to the line of sight (Figure 2), then (no matter how fast or slow) each next instant a new point on the continuous world line of the star is instantly reached and a last one is instantly passed. In between, each of the space-fixed points the star is passing across will just go to the very next further position on the plane, even, continuous and steady surface of the point star. In other words the shape in Figure 2 is instantly repeated and this repetition is, obviously, independent of the magnitude of the common non-zero velocity.

Anyhow, there are two boundary conditions for this recession mechanism to work. The first is that the perpendicular component of the velocity must be of a non-zero magnitude. The second boundary condition is that the magnitude must be big enough to dominate over all other local orbital speeds of the star relative to the observer.

\subsection{Recession as a function of the direction of velocity}

As shown earlier in Figure 4 for all directions of the velocity $\mathrm{V}$, except when exactly in the line of sight, there is a non-zero perpendicular component of $\mathrm{V}$ the magnitude of which is dependent on the angle $\theta$. But, as we have just seen, the recession rate and value are independent of the magnitude of the perpendicular component of velocity. Consequently the recession is independent of direction of the velocity.

But, same as for the magnitude of the velocity, for this recession mechanism to work the direction has also two boundary conditions to be fulfilled. The first is that the velocity is not completely in the line of sight. The second is that there must be some minimum angle $\beta$ for the perpendicular component of the velocity to dominate. So, for the observer in Figure 8 except when looking in the world line of the observer (the line in which the observer is moving along with the entire universe) and within the two narrow opposite conical zones around it, the recession rate is not a function of direction.

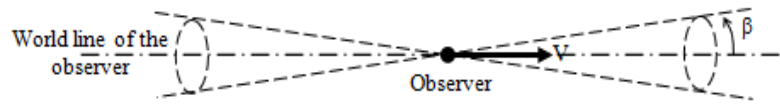

Fig. 8: Drawn by Author

\section{The moving universe and its agreement with some confirmed observations and find- ings}

Now, one can make a picture of the moving universe. The observer in Figure 9 is in the centre of a spherical universe (denoted by the black circle) that is entirely moving at a very high velocity $\mathrm{V}$.

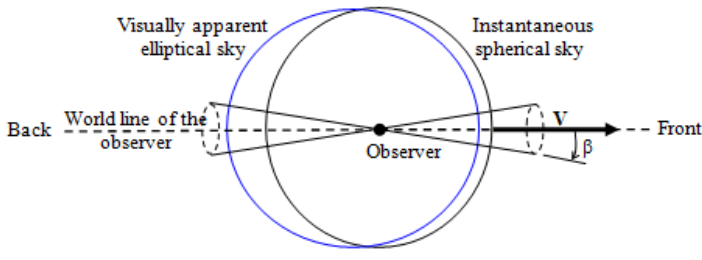

Fig. 9: Drawn by Author

By the kinematic postulate of the special theory of relativity (Skinner 1982), what appears to the observer is then the elongated universe denoted by the blue ellipse. The world line of the observer is the line in which the observer is moving along with the entire universe. The hemisphere in the direction of the velocity is the front and the opposite is the back sky. The angle $\beta$, as shown earlier, is the minimum angle for the perpendicular component of the velocity $\mathrm{V}$ to dominate. Now let us discuss the agreement of this entirely moving symmetrical universe with some known and confirmed observations and findings.

\subsection{Expansion and uniformity of the universe are both maintained}

From the previous discussion and in agreement with Edwin Hubble's findings it has been shown that in the entirely moving universe the recession is due to relative movement between the observer and the space-time fixed origins of the received pulses of light. This while there is no relative movement between the source and the observer. In other words the recession is taking place while the distance of the observed object from the observer is constant. So in this way the uniformity is undisturbed and also the observed apparent brightness and size of the stars remain steady and no stars will disappear due to this mechanism of recession.

\subsection{Olbers' paradox is escaped}

In the moving universe theory, unlike in the case of Newtonian expanding systems, galaxies are not flying away from the observer Nevertheless, the waves of light received from these galaxies, as already explained, are in concentric sets that are spreading out from their space-time fixed origins from which the moving observer is flying away. The observer is, hence, escaping the incandescent glow of Olbers' paradox.

\subsection{The southern sky is brighter and richer with nebu- lae and star clusters than the northern sky}

The southern sky is generally brighter than northern sky. Also, the southern sky has more number of star clusters and nebulae than the northern sky (Mayas 1999). Mayas attributed the first observation to the fact that the centre of the Milky Way, which is congested with a huge number of stars, is located in the southern sky (Mayas 1999). But Mayas gave no reasons for the second observation. In Figure 9 and due to the elongation that is caused by motion, the front sky is apparently closer and hence brighter than the back sky. Also, if we consider the black circle in Figure 9 as the edge of visibility, then it is evident that elongation of the apparent sky pulls in stars from beyond the visibility range in the front and pushes out others in the back sky.

\subsection{Powerful radiation in and around the world line of observer}

Cygnus A is one of the most powerful sources of radio waves (Inc. Staff Encyclopaedia Britannica Publishers 1979) and Cygnus X-1 is a powerful source of X-rays (Hawking 1995). Explanations given in the two references for the unknown sources of these powerful waves range from collision of two galaxies, explosion of stars to existence of black holes. In the moving universe theory, looking in the world line of the observer and within the two opposite narrow conical zones around it (Figure 8) there is no recession due to motion of the entire universe as shown earlier. So, Olbers' assumptions are no paradox in these two opposite zones. The assumptions hold. Hence, the radiation from within these two zones will be so powerful. A whole galaxy from beyond the edge of visibility or even a cluster of galaxies and any layers behind it falling within these two zones will be, with respect to observer, an invisible (fixed) source point of powerful radiation. Anyhow for this to be an explanation it is required that the location of these powerful sources is limited to the said zones.

\subsection{Shift of star position when observed during total eclipse of the sun}

Deflection of starlight is one of three classical observational tests of the general theory of relativity (Skinner 1982). Skinner stated: "the results of the three classical tests were believed to be in 
agreement with the predictions until recent evidence appeared that casts this agreement in doubt" (Skinner 1982, p. 321). It is no intension here to go into the details of the evidence or criticize the general theory of relativity. However, starlight deflection is again challenged by the following simple and reasonable alternative explanation of the observation that some stars are shifted from their known positions when observed during total eclipse of the sun. While orbiting around the sun our orbit, the sun and the star shown in Figure 10 are all moving at the velocity $\mathrm{V}$ along with entire universe.

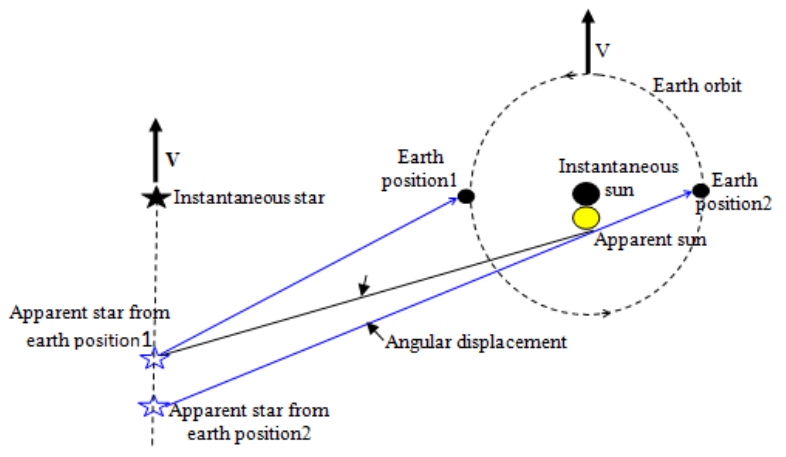

Fig. 10: Drawn by Author

The left hand side of the orbit is the season when this star can normally be seen. On the right hand side of the orbit the star can be seen only during total eclipse of the sun. As per the specia theory of relativity (Skinner 1982), the observer on our orbit cannot see the moving star at its instantaneous position (shown in black). The observer will see the historical apparent position of the star somewhere down on the world line of the moving star. The distance between the instantaneous and apparent positions of the star is equal to the magnitude of the velocity $\mathrm{V}$ times the time taken by light to travel from the star to the observer. This distance is, hence, directly proportional to the distance of the observer from the star. Now, from Earth position 1 the star is seen at its normal apparent position 1. During total eclipse of the sun the same star is seen from Earth position 2. But Earth position 2 is more distant from the star than Earth position 1 by almost the diameter of our orbit. Hence, the star apparent position 2 will be more distant from the instantaneous star than the normal apparent position 1 . Now it is evident that the angular displacement between the two apparent star positions as shown in Figure 10 is not due to starlight deflection. The observation that some stars are shifted from their known positions when observed during total eclipse of the sun stands as one of the strong proofs that the universe is entirely moving at some fantastic speed.

\subsection{Tide is more realistically explained}

Figure 11 shows $\mathrm{x}$-section of a simple tidal model of a planet of radius $\mathrm{R}$ covered uniformly with water. The planet is rotating at a constant angular speed $\omega$ around an axis normal to the XY coordinate system. The rotating planet and the XY coordinate system are all moving (along with the entire universe) at the velocity $\mathrm{V}$ in the positive direction of the $\mathrm{X}$-axis. As water is a real fluid, the inner layers of water on the surface of the solid planet will adhere to and rotate with the solid planet. The inner layers of water will hence cross the $\mathrm{X}$-axis and the $\mathrm{Y}$-axis at the maximum flow velocity $\omega \mathrm{R}$ Going outward each layer will tend to drive the layer above it by the viscous force of water. The layers will slip one above the other if some relative force (or momentum) is acting in a direction parallel to the layers (Massey 1971).

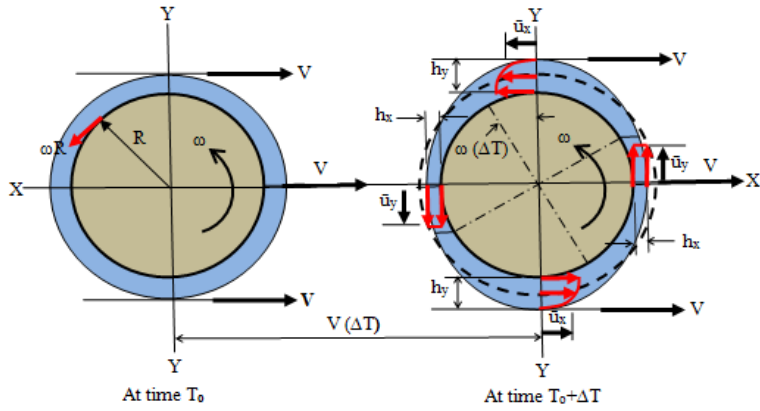

Fig. 11: Drawn By Author

Let us first consider the flow across the Y-axis. Now, for the actual radius of our planet, vast plane layers of water around the Yaxis are all moving at the very high velocity $\mathrm{V}$. The momentum acquired by these layers in the direction of the velocity $\mathrm{V}$ is hence so big that it resists the rotational movement induced by the rotating solid planet. So, this will produce velocity gradient of the flow of water driven by the rotating planet across the Y-axis. For the flow across the $\mathrm{X}$-axis the velocity $\mathrm{V}$ is acting normal to the layers of water and hence will not cause a velocity gradient. Due to the velocity gradient the mean velocity of flow $\overline{\mathrm{u}}_{\mathrm{x}}$ across the $\mathrm{Y}$-axis, is less than the mean velocity $\overline{\mathrm{u}}_{\mathrm{y}}$ across the $\mathrm{X}$-axis. By the continuity equation for incompressible fluids (Massey 1971) the volume flow rate $\mathrm{Q}$ across any $\mathrm{x}$-section normal to the direction of flow is constant. In equation form this can be expressed as follows:

$\mathrm{Q}=\overline{\mathrm{u}}_{\mathrm{y}} \mathrm{A}_{\mathrm{x}}=\overline{\mathrm{u}}_{\mathrm{x}} \mathrm{A}_{\mathrm{y}}$

Where, $A_{x}$ and $A_{y}$ are the areas of flow across the $X$-axis and the $\mathrm{Y}$-axis respectively.

Now, if we consider a water ring with width of unity, equation (7) will be:

$\mathrm{Q}=\overline{\mathrm{u}}_{\mathrm{y}} \mathrm{h}_{\mathrm{x}}=\overline{\mathrm{u}}_{\mathrm{x}} \mathrm{h}_{\mathrm{y}}$

Where, $h_{x}$ and $h_{y}$ are the heights of water flowing across the $X$ axis and the $\mathrm{Y}$-axis respectively.

But, (as explained above),

$\overline{\mathrm{u}}_{\mathrm{x}}<\overline{\mathrm{u}}_{\mathrm{y}}$.

From (8),

$\overline{\mathrm{u}}_{\mathrm{x}}=\overline{\mathrm{u}}_{\mathrm{y}} \mathrm{h}_{\mathrm{x}} / \mathrm{h}_{\mathrm{y}}$

From (9) and (10),

$\overline{\mathrm{u}}_{\mathrm{y}} \mathrm{h}_{\mathrm{x}} / \mathrm{h}_{\mathrm{y}}<\overline{\mathrm{u}}_{\mathrm{y}}$

From (11),

$h_{y}>h_{x}$.

Hence, there are two bulges and two depressions every one revolution as shown in Figure 11, which explains the two high and the two low tides in every 24 hours.

This explanation is more realistic for the following reasons:

1) As in reality, water is treated as a real incompressible fluid.

2) It is not necessary that the depth is uniform. Considering the real bathymetry of oceans, the un-even solid surface and the water contained within it will rotate as a solid surface and drive the layers of water above it.

3) In the above model if we consider a ring of land and water (as in reality) the explanation still holds. The water part of the ring will rise when passing across the $\mathrm{Y}$-axis and depress when passing across the $\mathrm{X}$-axis.

4) The model can also explain the terrestrial tides. The Earth crust is composed of plates that can slip above each other. When a plate is passing across the $\mathrm{Y}$-axis it will tend to keep 
moving in a straight line and in this way resist the rotational movement and hence cause some rise.

\subsection{Motion apparently elongates circular orbits while satisfying all of the three Kepler's laws}

The German astronomer Johannes Kepler (Williams 2003) deduced three laws that described the motions of planets around the sun:

1) The orbit of a planet around the sun is an ellipse.

2) The planet moves faster when closer to the sun and slower when distant.

3) The square of the period (in years) for one revolution about the sun equals the cube of the mean distance from the sun's centre, measured in astronomical units.

Assuming that our orbit is circular and the orbital speed is constant then the radius of the orbit is the mean distance from sun's centre and the period is the reciprocal of the constant orbital speed. This, hence, is the ideal case to satisfy the third law. In a moving universe the first and second laws are also satisfied. It is only when the velocity is normal to the plane of the orbit will then the visually apparent orbit remain circular but it will be enlarged as shown in Figure 12a. When the velocity is parallel to the plane of the orbit the apparent orbit will then be elongated by the maximum as in Figure 12b. When the velocity is inclined with respect to the plane then the vector sum of both effects will take place. So, like that, except when the velocity is exactly normal to the plane of the circular orbit, the visually apparent orbit is an ellipse which agrees with Kepler's first law.

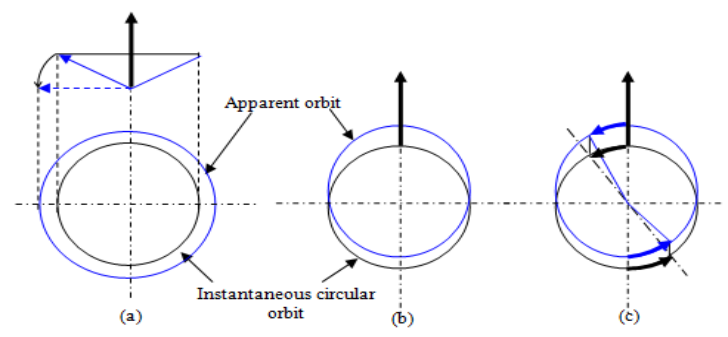

Fig. 12: Drawn by Author

In Figure 12c let us take two equal opposite angular displacements on the circular orbit (black arrows). At a constant orbital speed the two equal angular displacements mean two equal periods of time. The corresponding angular displacements on the apparent orbit (blue arrows), are simply obtained by shifting the tail and the head of each black arrow in the direction of the velocity V. As shown in Figure12c, this shift will make the arc angle increase when moving towards the centre of the circular orbit (the sun) and decrease when moving away. So, on the apparent orbit and for the two equal periods of time, the angular displacement is bigger when closer to the sun and smaller when distant. This, hence, satisfies Kepler's second law.

\subsection{Eccentricity of elliptical orbit can be expressed in terms of the velocity ( $V$ ) and the speed of light}

Now considering the simple case when the velocity $\mathrm{V}$ is parallel to the plane of a circular orbit of radius R (Figure 13) we can describe the resulting apparent elliptical orbit as follows:

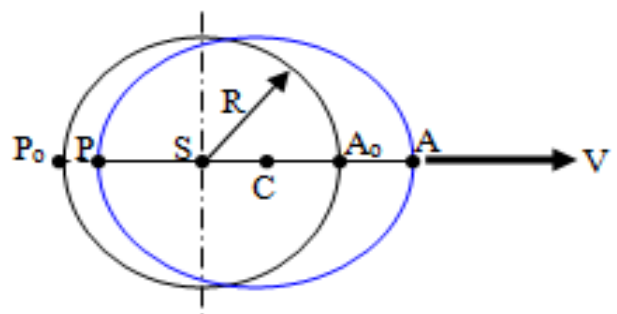

Fig. 13: Drawn by Author
The centre of the circular orbit $S$ is the focus of the apparent orbit. The size of the apparent orbit is the periapsis distance SP. The apoapsis distance is SA. The semi-major axis AC, CP is half the long axis AP. Elongation or eccentricity (e) is the ratio CS/CP (Williams 2003). Now, on the circular orbit of radius $R$ that is entirely moving at the velocity $\mathrm{V}$, points $\mathrm{A}_{\mathrm{o}}$ and $\mathrm{P}_{\mathrm{o}}$ are apparently at $\mathrm{A}$ and $\mathrm{P}$ respectively. It is interesting to show here that the eccentricity is also equal to the ratio $(\mathrm{V} / \mathrm{c})$ where (c) is the speed of light as follows:

Let $(\Delta \mathrm{T})$ be the time taken by light to travel from $(\mathrm{S})$ to $(\mathrm{P})$ and for the planet to move from $\mathrm{P}_{\mathrm{o}}$ to $\mathrm{P}$

Then:

$\mathrm{SP}=\mathrm{c}(\Delta \mathrm{T})$

And,

$P P_{o}=V(\Delta T)$

But from Figure 13,

$\mathrm{SP}+\mathrm{PP}_{\mathrm{o}}=\mathrm{R}$

From (13), (14) an (15),

$\Delta \mathrm{T}=\mathrm{R} /(\mathrm{c}+\mathrm{V})$

Hence from (13) and (16),

$\mathrm{SP}=\mathrm{R} /(1+\mathrm{V} / \mathrm{c})$.

In same way it can be deduced that:

$\mathrm{SA}=\mathrm{R} /(1-\mathrm{V} / \mathrm{c})$

$\mathrm{CP}=\mathrm{AC}=(\mathrm{SP}+\mathrm{SA}) / 2$.

$\mathrm{CP}=\mathrm{R} /(1+\mathrm{V} / \mathrm{c})(1-\mathrm{V} / \mathrm{c})$

$\mathrm{CS}=\mathrm{CP}-\mathrm{SP}$.

$\mathrm{CS}=(\mathrm{V} / \mathrm{c}) \mathrm{R} /(1+\mathrm{V} / \mathrm{c})(1-\mathrm{V} / \mathrm{c})$

$\mathrm{e}=\mathrm{CS} / \mathrm{CP}=\mathrm{V} / \mathrm{c}$

When the velocity $\mathrm{V}$ is inclined at angle $(\varnothing)$ with respect to the plane of the circular orbit, the velocity $\mathrm{V}$ will then be substituted with $\mathrm{V} \cos \varnothing$ and then the eccentricity will be:

$\mathrm{e}=(\mathrm{V} / \mathrm{c}) \cos \varnothing$.

From the data given by Mayas, the eccentricity of our orbit is equal to 0.01672 , (Mayas 1999) then:

$\mathrm{e}=(\mathrm{V} / \mathrm{c}) \cos \emptyset=0.01672$.

Hence,

$\mathrm{V} / \mathrm{c}=0.01672 / \cos \varnothing$.

Hence, 
$\mathrm{V} / \mathrm{c} \geq 0.01672$

This means that if the eccentricity of our orbit is purely due to movement at the velocity $\mathrm{V}$, then the magnitude of this velocity is equal to or greater than $1.672 \%$ of the speed of light.

\subsection{Though expansion is speeding up, the universe is possibly flat}

Recent results from three different telescopes, as per Sincell, confirmed that the universe is flat (Sincell 2001). For the universe to remain flat the total density of matter and energy must be exactly equal to a certain critical value which is determined by the rate of expansion. To determine the rate of expansion, Sincell reported that two independent teams, Saul Perlmutter and his team at the Lawrence Berkeley lab in California and Brian Schmidt's team at the Harvard University Centre for Astrophysics, started weighing the universe in 1993. To weigh the universe, both teams searched digital images of large swatches of sky for the sudden appearance of exploding sun-like stars called Type 1a supernovae. A special property of these supernovae as reported by Sincell, is that within a week of exploding and before fading into obscurity months later, all Type 1a supernovae reach the same peak luminosity. This special property helped to calculate the exact distance to each Type 1a supernova. Follow-up pictures from a worldwide array of telescopes reveal the colour of the fading supernova flash, which was used to determine the rate of expansion. It was a big surprise then to find that the expansion of the universe was speeding up (Sincell 2001). Under the gravitational effect of all matter and energy in the universe this is not supposed to happen as per Sincell. In the moving universe theory the recession revealed by the light spectrum, as shown earlier, is continuously repeated and can be instantly observed while the distance between the observer and the point star is constant. So, the finding that the universe is flat is possible. The actual Newtonian rate, which cannot be revealed by spectroscopy, may be exactly equal to a critical rate that is not determined yet. The other recent finding that the expansion of the universe is speeding up can also be explained as follows:

The recession rate of any single space-fixed point within a point star in the entirely moving universe is actually increasing with time as diagrammatically shown earlier in Figure 6. This speeding up recession can also be found by differentiating the distance with respect to time as follows:

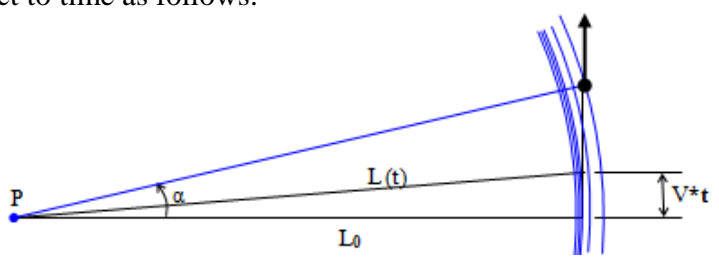

Fig. 14: Drawn by Author

In Figure 14 shown above, let $\mathrm{L}_{0}$ be the initial minimum distance of the observer from point $\mathrm{P}$. At any point of time $t$ the distance moved by the observer is $\mathrm{V} * \mathrm{t}$. At this point the distance of the observer $\mathrm{L}(\mathrm{t})$ from point $\mathrm{P}$ can then be written in the form:

$\mathrm{L}(\mathrm{t})=\left(\mathrm{L}_{\mathrm{o}}^{2}+\mathrm{V}^{2} * \mathrm{t}^{2}\right)^{1 / 2}$.

The rate or velocity of recession $u$ is the first derivative of the above equation:

$\mathrm{u}=\mathrm{dL} / \mathrm{dt}=(1 / 2)\left(\mathrm{L}_{0}^{2}+\mathrm{V}^{2} *^{2}\right)^{-1 / 2}\left(2 \mathrm{~V}^{2} * \mathrm{t}\right)$.

Hence,

$\mathrm{u}=\mathrm{V}^{2} /\left(\mathrm{L}_{0}^{2} / \mathrm{t}^{2}+\mathrm{V}^{2}\right)^{1 / 2}$.

Hence the rate or velocity of recession as shown by the above equations is speeding up. But as explained earlier at any instant the observer simultaneously receives light from all points the point star is simultaneously passing across. So, at any instant we examine the spectrum all rates from the minimum to the maximum are there as far as the star is plane, even, continuous and steadily bright. The rate of recession of a single space-fixed point is, however, possible to find when some change is detected e.g. fading out of a star. Now, as stated earlier, the eye records all the light that is received simultaneously, even though, if the object is extended, this light was emitted at different times from parts of the object at different distances from the observer (Skinner 1982). Based on this statement it can also be said that, light that is simultaneously emitted from parts on an extended object at different distances from an observer will not be simultaneously received by the observer. This light will be received in the order of distances from the shorter to the longer. The fading out of Typela supernovae as per Sincell, was a change that was followed up from the time just before taking place and during the change to obscurity months later (Sincell 2001). So in the entirely moving universe and during change, any state of change will be simultaneously emitted from all the space-fixed points the point star is simultaneously passing across. This simultaneous state of change will, hence, be received by the moving observer in the order of distances from the shorter to the longer as shown by colour change in Figure 15 below.

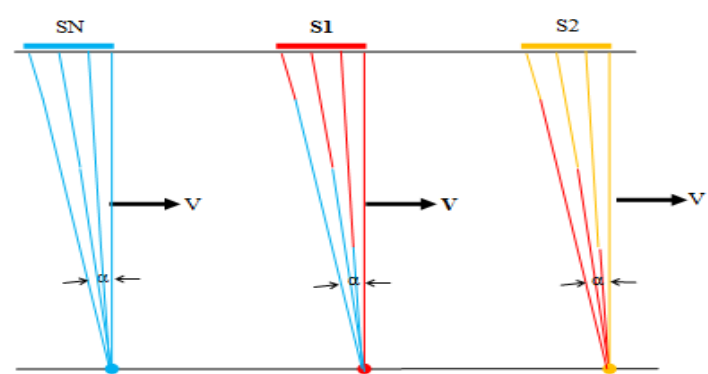

Fig. 15: Observation during change of a Star in an entirely moving universe: $\mathrm{V}=$ Velocity of the entire universe, $\alpha=$ The point star constant of proportionality, $\mathrm{SN}=$ Normal state of the star, $\mathrm{S} 1=$ First simultaneous state of star change, $\mathrm{S} 2=$ Second simultaneous state of star change. (Drawn by Author).

So, this is same as observing recession of a single space fixed point in the entirely moving universe which, as shown above, is speeding up with time. This, hence, explains the above finding that the expansion of the universe is speeding up.

\section{Conclusion}

As concluded from the discussion: For an observer in an entirely moving universe looking in any direction (except in two narrow opposite conical zones around the world line of the observer) at a distant object then: 1) When the object reaches a new point on its continuous world line that point will become a space-fixed origin of concentric waves of light and it will recede relative to the observer at an speeding up rate from an initial minimum to a final maximum distance within the same line of sight 2) The observer will simultaneously receive all the recession values from the minimum to the maximum. 3) The maximum value of recession $\Delta \mathrm{L}$ is directly proportional to the initial distance between the object and the observer. 4) This recession is continuously repeated and can be instantly observed while the mean distance between that object and the observer remains same.

What remains now is how fast the universe is moving and in which direction. Some indications of the direction and the magnitude of the velocity can be primarily deduced as follows:

1) The fact that the southern sky is brighter and having more number of nebulae and star clusters than the northern sky, indicates that the direction is somewhere to the south with respect to our planet.

2) As shown earlier, if the elongation of our orbit is completely due to movement of the universe then the eccentricity, $(\mathrm{V} / \mathrm{c})$ 
$\cos \varnothing$ is equal to 0.01672 . It means that the magnitude of the velocity $\mathrm{V}$ is equal to or greater than $1.672 \%$ of the speed of light.

3) Star shift during total eclipse of the sun, which is now believed to be better explained by the moving universe theory, can also help in finding the magnitude and direction of the velocity V.

4) The world line of our planet can be found by looking for distant stars from which light spectra do not show recession.

\section{References}

[1] Hawking, S. (1995).A brief history of time. Toronto: Bantam Books.

[2] Inc. Staff Encyclopaedia Britannica Publishers (1979).Energy the fuel of life. GB. Bantam Britannica Books.

[3] Massey B. S. (1971).Mechanics of fluids 2nd edn. Van Nstrand Reinhold. London.

[4] Mayas E. (1999) Encyclopedia viewing macroscopic: the universe Lebanon Library Publishers.

[5] Sincell M (2001) A new beginning. Times Mirror Magazines, Popular Science, February 2001, 70-74.

[6] Skinner, R. (1982). Relativity for scientists and engineers. New York: Dover Publications.

[7] Williams, J. "Orbit." Microsoft Encarta Encyclopaedia Deluxe 2003. Web. 